\title{
A Practical Synthesis of Dynamic Role Settings in Telecare Services
}

\author{
Ying Liu, Jean Bacon \\ Computer Laboratory, University of Cambridge \\ JJ Thomson Avenue, Cambridge CB3 OFD, UK \\ email: firstname.lastname @ cl.cam.ac.uk
}

\begin{abstract}
Role provisioning is an essential yet complex aspect of the design of virtual organisations. This paper addresses an even more complex scenario, that of role provisioning in telecare. Here the roles may be doctor, nurse, carer, etc. And they must be enabled to participate in monitoring remote care-receivers via the internet, using their professional expertise and skills, with access to online-resources. We show that the complexity of role provisioning is attributable not only to defining a role or roles within an organisation, but also to a large degree of uncertainty on how to manage online-contexts. We illustrate this by giving practical scenarios including services in on/offline mode, patient participation, and professional participation with different care-levels. We call these online-contexts "dynamic role settings". This work describes an approach to establishing a dynamic channel of interaction between a care-provider (e.g. doctor), necessary resources, and a care-receiver (e.g. patient), so as to manage the care-receivers' needs and the care-providers' responsibilities.
\end{abstract}

Telecare; e-Service; Role Provisioning; Dynamic Trust; Role Context; Dynamic Access; RBAC; Meta-synthesis.

\section{INTRODUCTION}

Role provisioning is a process to define, implement, set up, and activate an access control system based on the idea that users are typically granted access to resources according to their roles in an organisation. Rules are set up for, e.g. role assignment, role authentication and action authentication [2][10][14].

Although role provisioning is understood to some extent, it is widely acknowledged as being one of the most difficult, complex and imperfect processes in the design of computing network applications and services ${ }^{1}$. Moreover, additional complexities are emerging from applications of new technologies, integrated via the Internet, notably,

\footnotetext{
1 Microsoft .NET Customer Solution Case Study, http://download.microsoft.com/documents/customerevidence/20836_A zMan_Case_Study_Lighthouse_Final.doc
}

wireless sensor technologies ${ }^{2}$, hand held computing, sensor networks, data communication networks, mobile and wearable devices ${ }^{3}$. To design an application in such a context, role provisioning becomes broader than protecting access to the system and data. Trust in the behaviour and competence of a principal acting in a role becomes of greater concern, as does making available sufficient resources in a timely manner for a required task [3]. Thus, role provisioning must be synchronized with provisioning resources that may be needed by the user.

In e-commerce, issues of trust are affecting online communities such as eBay, where resources include buyers, sellers, suppliers, consumables, and multiple data sources. A given use of resources can be rated according to a set of past transactions in the community [20]. The availability and reliability of a service may rely on community members' recommendations, reputation, agreements and contracts, or on brand names, individual experience, knowledge and risk taking. However, the use of telecare is completely different from that of eBay, being defined as "the use of information and communication technologies to transfer medical information for the delivery of clinical services to patients in their place of domicile." [21]

Telecare applications have been developed for wireless networks ${ }^{4}$. Stankovic et al. investigate the potential and challenges of using wireless sensor networks for in-home healthcare [17]; Medixine offers a diabetes self-monitoring solution integrating blood glucose measurements [12]; The TPAS system (Think Positive Asthma System) combines a peak flow device with mobile technologies and generates a self-care plan that is agreed between an individual and a GP [11]. Several applications are deployed, e.g. the Keelung community - based integrated screening [7], which uses modern transmission of glucose values to reduce the need for clinic visits, and thus the cost of treatment [6]; A multi-site, telemedicine service for emergencies [19]; A use

\footnotetext{
2 NIST Wireless Ad Hoc Networks Links, http:/ / www.antd.nist.gov/ wctg/ manet/ adhoclinks.html 3 About Smart Phones, http:/ / www.about-smart-phones.com

4 Super Paper: Medical Applications of Wireless Networks, http://userfs.cec.wustl.edu/ in1/draft_cse574.html
} 
of $3 \mathrm{G}$ mobile phone links for consultation between a moving ambulance and a hospital base station [4]. To fully realise the potential of telecare, role provisioning must be possible in real time and must achieve (and be perceived as achieving) responsibility, availability and reliability in consulting, diagnosing or providing a prognosis [21][22][23]. Also, patients' details must be secure, and inaccessible to those unauthorized. A large variety of approaches, methods and techniques exist, which indicates the complexity of setting up roles in these contexts, e.g. [23][24].

In this paper, we examine roles' dynamic contexts in a service environment. In section 2 we briefly review our hypothetical environment in terms of physical infrastructural elements and service types. In section 3 we illustrate practical scenarios of roles' dynamic settings such as services in "on/offline service mode", "patient participation", "care professional participation", and "care-levels". Finally, we give conclusions in section 4 and raise key research questions in section 5 .

\section{HYPOTHETICAL SERVICE ENVIRONMENT}

A service is provisioned in order to give value to a client, for example, to book a train ticket on a given date. In this case, the service one seeks is the provision of a train ticket (entity) that is to be used within the specified parameters. Here it is defined as a computational entity, e.g. for accessing a timetable, receiving an electronic train ticket and making a payment over the internet. It addresses a need at a specific point in time - what we need, when we need it and wherever we need it via any device we choose and access.

A web service is a means by which software components can be published, discovered and invoked over the network using standards [5][16][9]. Service Oriented Computing [15] has been widely adopted as a new paradigm, and there are different scales in building computational entities to manage and distribute the services [1]. Not only are the services required to be easy to use, and personalised or context-aware while in use [8], but also, most importantly, must be secure and trusted enough for users [2][3]. Telecare services generally have all these features.

But unlike current services via the web which are mainly developed for reading textual information (e.g. train timetable, list of costs), telecare services include an increasing number of sensory devices and mobile ICT systems with greater specialisations. The services are driven by the settings where the devices are used and where the data are acquisitioned, processed and distributed.

Here we are concerned with such a system, see Figure 1 , that has devices measuring physiological status and other data. It also has components that present this data to individual users. The network elements transmit data to a service centre and then to a health professional (e.g. physician) for review. This system will have a profound impact on the type, content, location, operation and functionality of care products and services.

\section{A. Physical Infrastructure}

As Figure 1 illustrates, the infrastructure consists of a wireless body sensor network for health monitoring, which is integrated with a broad multi-tier telecare solution-architecture. Telecare spans a network comprising individual health monitoring systems that connect through the internet to a medical server, shown on the right. The top tier has mobile connections. The middle tier is a user centred service environment with trust, and the lower tier encompasses a home based data communication network. Users may wear a number of sensor nodes which sample vital functions and transfer the relevant data to a personal server through a wireless personal network. This data connection may be implemented using ZigBee (802.15.4) or Bluetooth (802.15.1). The personal server, which could be implemented on a personal digital assistant (PDA), cell phone, or home personal computer, sets up and controls the sensor network, provides a graphical or audio interface to the user, and transfers the information about health status to the medical server through the Internet or mobile phone networks (e.g. GPRS, 3G).

The medical server keeps electronic medical records of registered users and provides various services to users, medical personnel, and care providers. It is the responsibility of the medical server to authenticate users, to accept health monitoring session uploads, to format and insert this session data into corresponding medical records, to analyse the data patterns, to recognize serious health anomalies in order to contact emergency care providers, and to forward new instructions to users such as physician prescribed exercises. The patient's doctor or physician can access the data from his/her office via the Internet or handheld devices and examine the data to ensure that the patient is within expected health metrics (heart rate, blood pressure, activity); that the patient is responding to a given treatment; or that the patient has been performing the given exercises.

A server agent may inspect the uploaded data and create an alert in the case of a potential medical condition. Data must be acquired and uploaded to the server. A message must then be sent that data is available, and the suspected medical condition.

The responsible health professional(s) must review the data and make clinical decisions, e.g.

- immediate referral for treatment,

- urgent diagnosis by a doctor,

- non-urgent, continued observations,

- intervention by a doctor.

- no decision needed, and/or

- no action needed. 


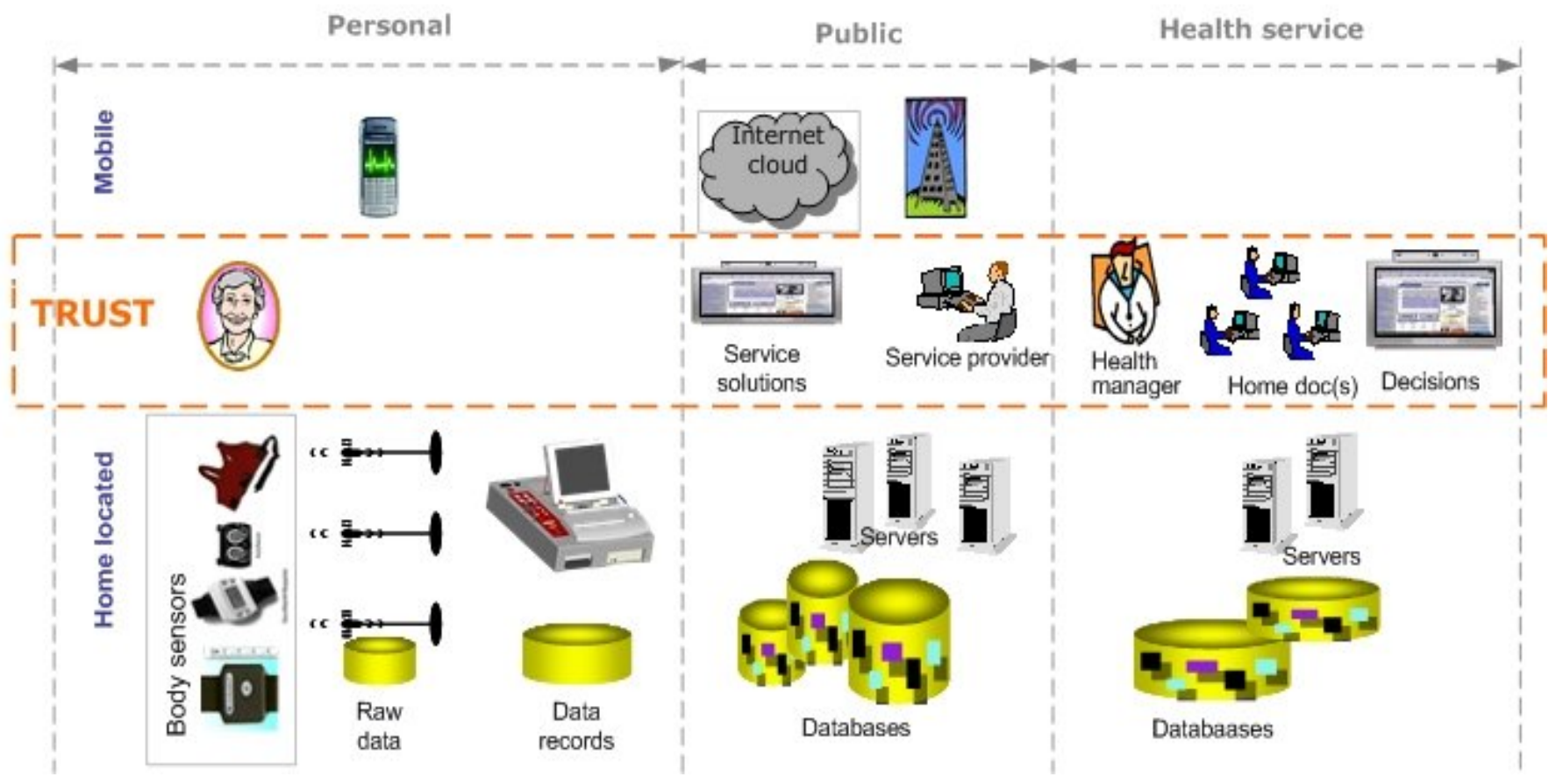

Figure 1. Service physical infrastructural elements

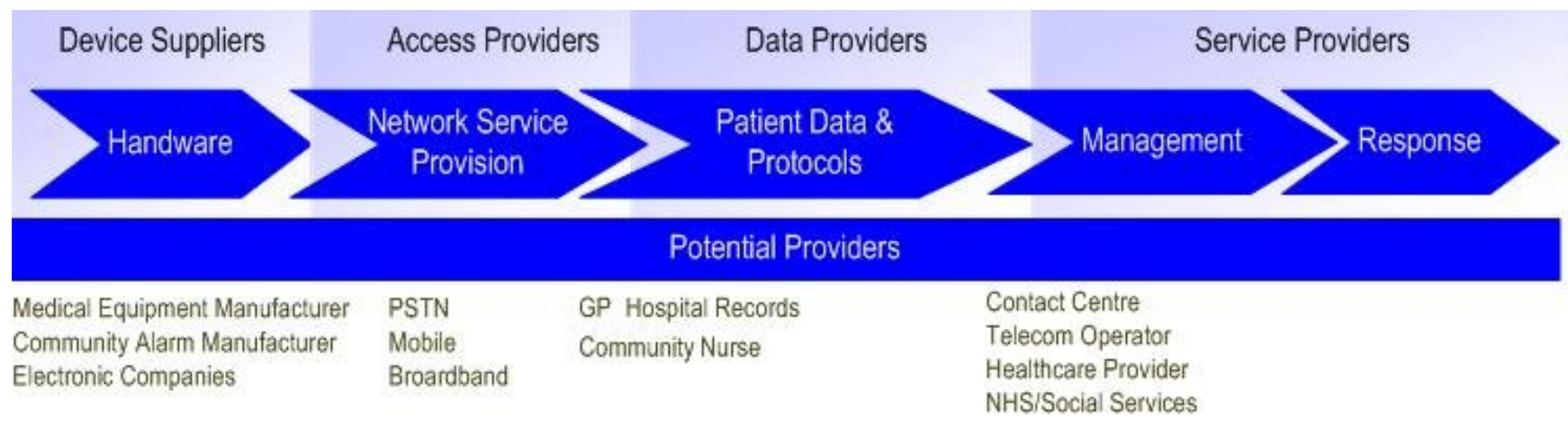

Figure 2. Service providers

TABLE I. INSTANCES OF SERVICE TYPES

\section{Telecom service}

Calling card, Call forwarding, Call screening, Broadband, VPN

\section{Telecare services}

Booking service, Reminders, Emergency response, Online consultation, Online care, Health warning, Daily living monitoring, Physiological monitoring, Vital sign monitoring, Condition specific monitoring. 


\section{B. Service Types}

The service type provided by a conventional telecommunication network is evident - it is driven by familiar tele- or mobile-phones, and it moves bits from one place to another. For most of the last century there was really only one range of terminal device (the telephone). But very soon the developments in devices that present services to users will increase considerably, see Table 1 . There have already been various types of device that need better network support, so as to link us to a dedicated service space. For a wireless sensor network, moving bits is only a means to an end, since the end users need high-level information derived from the raw data. Additionally, many concepts, such as scoping interactions to specific locations or to time intervals, will become important. Service participants may include device suppliers, access providers, data providers and service providers, as Figure 2 shows.

\section{DYNAMIC ROLE SETTINGS}

In the environment described above, named roles such as patients, doctors or other health professionals are in static settings (e.g. system administrators, clinical coders, or health record registrars). There is a growing awareness of the importance of dynamic roles, because many roles cannot be defined in the absence of certain online dynamic contexts.

\section{A. Role's Dynamic Interactions}

We give the following examples to illustrate how roles may be embedded in a number of parameterised building-blocks (noted by <--> hereafter) which can be combined to create an end-user service model.

<on/offline-mode> An instance of a service can be set up to operate in real-time or store-and-forward mode. This is because there may be different needs for personnel and resource management. The parties concerned must be scheduled, and it is likely that several healthcare professionals are involved in a clinical query. Also, when a patient has difficulty in communicating, both the local carer and the remote physician may need to be available at the same time (in online mode). In contrast, store-and-forward mode is an asynchronous interaction, so that a clinical query, for example, can be transmitted and answered by a specialist at a convenient time previously agreed. As an example, a global standard for DICOM $^{5}$ (Digital Imaging Communications in Medicine) enables the development of image delivering, processing and viewing systems. Asking for a second opinion on a particular image is common practice in radiology. An offline mode service supports asynchronous consultation of a remote specialist.

<participation> is set by on/offline modes, location identification, registration, enrolment, personal

\footnotetext{
5 http://medical.nema.org/
}

identification, admission, appointment, and schedule. Thereby healthcare service providers can set up new referrals for participants, e.g., patients and care-professionals.

<patient-participation> sets up the location for a patient "visit" as the home, a care centre, indoors or outside. For example, a patient may need to be seen in person for their first visit, then have the option of home or a care centre for subsequent visits, if they are close to a facility equipped for further required tests. It can also ensure an annual personal visit to a care centre with telemedicine for interim "visits" every three to six months.

<care-professional-participation> can be set up in offline mode and indicate a time period within which a visit must be made, e.g. when a radiologist wants to sell an after-hours diagnostic interpretation service for small hospitals and healthcare centres. The radiologist may visit them, say, every third week to perform ultrasound studies and diagnose some of the imaging studies performed by the local personnel. The radiologist is likely to diagnose only a fraction of the cases that a general practitioner has considered; only those selected as requiring a specialist's review.

\section{B. Role's Care Levels}

We may have the following software building blocks indicating care levels.

<care-level-indicator> assigns a degree of care responsibility and a scale of professional process. These include ambulatory care, primary care, clinical care, and critical care. "health-and-wellness" can be in primary care; "care-monitoring" can be in ambulatory care or clinical care; "clinical-management" can be in a combination of all these. Four scales of professional processes are essential.

<primary-care-process> requires care to be given in a well organised, but routinely managed process-unit with guidelines. In a typical care monitoring service under primary care providers within a general clinical practice, physicians may not often directly involve patients but may still enhance care.

<medical-process> requires care to be given in specific clinical conditions, e.g. diagnosis of dermatological problems, diabetes management, etc.

<specialist-process> requires communication among specialists for assessment, diagnosis and joint decisions to be made. This process is multi-disciplinary and time and location sensitive, such as pre- and post-mission, daily- or morning, sit-down versus bedside.

<emergency-process> requires immediate diagnosis and treatment of acute illnesses and injuries.

\section{Resourcing Roles}

Resources-management uses entities contained in templates (see section 3.A \& 3.B) as operational contexts to 
search or create directories, repositories, and/or indexes for, e.g. customer subscriber management, online personnel healthcare management. Thereby it may be possible

- to create a certification, delivered by an external certification team,

- to follow self-assessment against functional requirements and validation of electronic data messages, and

- to automatically make appointments or request medication renewals.

<virtual-human-resources-management> uses directories for consistent, uniform management and jurisdiction when exchanging information, e.g. certifying personnel, partner, organisation capabilities who meet the functions and specifications. E.g., "backup-of-role", "decentralisation-of-authority", "collaboration-of-work" can be created at run-time.

<backup-of-role> When an individual is on a business trip or long-term absence, the job functions need to be maintained by others. This requires that somebody be delegated the authority to do the absent individual's job.

<decentralisation-of-authority> When an organisation needs to set up initially, or reorganise subsequently, job functions are re-distributed.

<collaboration-of-work> When people need to collaborate with others in the same organisation or other organisations, some access authorities are granted to enable sharing-information.

\section{CONCLUSIONS}

In a telecare service environment, complex roles with notions of trust must be satisfactorily pursued. We have analysed some telecare online dynamic contexts in order to investigate and set roles. We show that service providers contract with end-users; and other participants are sub-contracted with respect to services. A meta-synthesis approach enables us to identify roles' environments with aspects of trust, and systematically address the services as well as examine important assumptions about them. Role provisioning is a service commitment to trustworthiness. Roles have to be set up through a service platform (static environment). Roles must be provisioned, activated and enabled in dynamic settings where telecare service operations take place, and be associated with trust qualification and quantification.

\section{DISCUSSION}

We need to define roles as transactions from different domains when constraints have to be treated according to individual factors. The ideas are to map roles and roles' functionalities through provisions for anonymity, entity naming, resource requirements, history and profile assessment for storing reputations and the behaviour of recommendation protocols.

We need to further model dynamic role settings with a focus on the following aspects:

- Roles have functions and responsibilities, but when and how should they be trusted?

- There are transient, role-based responsibilities in managing and maintaining the integrity of resources including data and actors. How should such policies be validated?

\section{ACKNOWLEDGEMENT}

We gratefully acknowledge the support of EPSRC through the CareGrid grant EP/C53718, and many discussions with project researcher Jat Singh and project co-investigator Dr. Ken Moody at Opera Group, Computer Laboratory, Cambridge University.

\section{REFERENCES}

[1] J. Bacon, "Expectations and reality in distributed systems," in Proceedings of IASTED International Conference on Parallel and Distributed Computing and Networks, Insbruck, Austria, February 2005, pp.vii - xiv.

[2] J. Bacon and K. Moody, "Adaptive middleware: toward open, secure, widely distributed services," Communications of the ACM, vol. 45, no. 6, June 2002, pp. 59-63.

[3] J. Bacon, K. Moody and W. Yao, "A model of OASIS role-based access control and its support for active security," ACM Transactions on Information and System Security, vol. 5, no. 4, November 2002, pp. 492-540.

[4] K. A. Banitsas, K. Perakis, S. Tachakra and D. Koutsouris, "Use of $3 \mathrm{G}$ mobile phone links for tele-consultation between a moving ambulance and a hospital base station," Journal of Telemedicine and Telecare, vol. 12, no. 1, 2006, pp. 23-26.

[5] E. Cerami, Web Services Sssentials, O’Reilly, 2002.

[6] H. P. Chase, J. A. Pearson, C. Wightman, M. D. Roberts, A. D. Oderberg, A.D and S. K. Garg, "Modern transmission of glucose values reduces the costs and need for Clinic Visits," Diabetes Care, vol. 26, 2003, pp. $1475-1479$.

[7] Y-H. Chiu, L-S. Chen, C-C. Chan, D-M. Liou, S-C. Wu, H-S. Kuo, H-J. Chang and T-H. Chen, "The information system for community-based multiple screening in Keelung, Taiwan," International Journal of Medical Informatics, vol. 75, May 2006, pp.369-383.

[8] P. L. Emiliani and C. Stephanidis, "Universal access to ambient intelligence environments: opportunities and 
challenges for people with disabilities," IBM Systems Journal, vol. 44, no. 3, 2003, pp. 605 - 619.

[9] D. Fensel, U. Keller, H. Lausen, A. Polleres and I. Toma, I, "WWW or What's Wrong with Web service discovery," Proceedings of Workshop on Frameworks in Web Service, Innsbruck, Austria, June 2005.

[10] D. Ferraiolo, D. R. Kuhn and R. Chandramouli, Role-Based Access Control, Artech House, 2003.

[11] D. Ryan, W. Cobern, J. Wheeler, D. Price and L. Tarassenko, "Mobile phone technology in the management of asthma," Journal of Telemedicine and Telecare, vol. 11, 2005.

[12] A. J. Farmer, O. Gibson, C. Dudley, K. Hannaby, P. Hayton, L. Tarassenko and H. A. W. Neil. "A randomised controlled trial of the effect of real-time telemedicine support on glycemic control in young adults with type 1 diabetes," Diabetic Medicine vol. 22, no. 2, 2005, pp.82-83.

[13] J-F. Gu and X-J. Tan, "A test on meta-synthesis system approach to forecasting the GDP growth rate in China," Proceedings of the 47th Annual Meeting of the International Society for the Systems Sciences, Hersonissos, Crete, 6-11 July 2003.

[14] A. Hammerle, A. Karageorgos, M. Pirker, A. Reitbauer and G. Weichhart, (2004), "A role-based infrastructure for customised agent system development in supply networks," Proceedings of Systems, Man and Cybernetics, 2004 IEEE International Conference, vol.5. 10-13 October 2004, pp.4692 - 4699.

[15] M. N. Huhns and M. P. Singh, "Service oriented computing: key concepts and principles," IEEE Internet Computing, vol. 9, Issue 1, Jan-Feb 2005, pp. $75-81$.

[16] C. Preist, "A conceptual architecture for semantic web services". Proceedings of the International Semantic Web Conference (ISWC), 7-11 November 2004.

[17] J. A. Stankovic, Q. Cao, T. Doan, L. Fang, Z. He, R. Kiran, S. Lin, S. Son, R. Stoleru and A. Wood, "Wireless sensor networks for in-home healthcare: potential and challenges," Proceedings of High Confidence Medical Device Software and Systems (HCMDSS) Workshop, Philadelphia, PA, USA, 2-3 June 2005.

[18] X-J. Tang and Y. Liu, "Computerized support for qualitative meta-synthesis as perspective development for complex problem solving," Proceedings of International Conference on
Creativity and Innovation in Decision Making and Decision Support (CIDMDS 2006), LSE. London.

[19] K. Waite, F. Silver, C. Jaigobin, S. Black, L. Lee, B. Murray, P. Danyliuk and E. M. Brown, "Telestroke: A multi-site, emergency-based telemedicine service in Ontario," Journal of Telemedicine and Telecare, vol 12, no. 3, 2006, pp.141-145.

[20] L. Xiong and L. Liu, L, "A peputation-based trust model for peer-to-peer ecommerce communities," Proceedings of IEEE Conference on E-Commerce (CEC’03), 2003.

[21] A.C. Norris, Essentials of Telemedicine and Telecare, Willey. 2002.

[22] S. A. Nancarrow and A. M. Borthwick, "Dynamic professional boundaries in the healthcare workforce", Sociology of Health \& Illness, vol.27, no.7, 2005, pp.897-919.

[23] L. Zhang, G. Alm and B. Chu, "A role-based delegation framework for healthcare information systems," Proceedings of the $7^{\text {th }}$ ACM Symbosium on Access Control Models and Technologies, Monterey, California, USA. 2002, pp.125-134.

[24] F. Hansen and V. A. Oleshchuk, "Location-based security framework for use of handheld devices in medical information systems," Proceedings of Fourth Annual IEEE International Conference on Pervasive Computing and Communications Workshops, Pisa, Italy, 2006, pp.565-569.

[25] S-H. Chae, W. Kim, D-K. Kim, “uT-RBAC: Ubiquitous Role-Based Access Control Model”, IEICE Transactions on Fundamentals of Electronics, Communications and Computer Sciences E89-A(1), 2006 pp. 238-239. 